17 Anderson, J. R., Goudie, R. B., and Gray, K. G., British fournal of Experimental Pathology, 1960, 41, 364.

18 Fulthorpe, A. J., et al., Fournal of Clinical Pathology, 1961, 14654.

19 Holborow, E. J., et al., British Fournal of Experimental Pathology, 1959, 40, 583.

20 Evered, D. C., et al., British Medical fournal, 1973, 1, 657.
21 Temple, R., et al., Mayo Clinic Proceedings, 1972, 47, 872.

${ }^{22}$ Carlson, H. E., Temple, R., and Robbins, J., fournal of Clinical Endocrinology and Metabolism, 1973, 36, 1251.

${ }^{23}$ Cotton, G. E., Gorman, C. A., and Mayberry, W. E., New England fournal of Medicine, 1971, 285, 529.

24 Utiger, R. D., Fournal of Clinical Investigation, 1965, 44, 1277

\title{
Clobetasone Butyrate, A New Topical Corticosteroid: Clinical Activity and Effects on Pituitary-Adrenal Axis Function and Model of Epidermal Atrophy
}

\author{
D. D. MUNRO, LYN WILSON
}

British Medical fournal, 1975, 3, 626-628

\section{Summary}

Clobetasone butyrate is a new corticosteroid, selected for study because of its combination of good activity in the vasoconstriction test and low systemic activity in animals. Formulated as an $0.05 \%$ ointment and cream (Molivate) it was clinically effective in patients with eczema, its activity being significantly greater than that of hydrocortisone $1 \%$ or fluocortolone $\mathbf{0 . 2 \%}$ (Ultradil). Under conditions that predispose to maximal percutaneous absorption clobetasone butyrate ointment had minimal effect on hypothalamic-pituitary-adrenal function. In an animal model of cutaneous atrophy it caused less thinning of the epidermis than steroids other than hydrocortisone. Clobetasone butyrate $0.05 \%$ ointment and cream gave every indication of offering clinically effective topical anti-inflammatory activity with a wide margin of safety.

\section{Introduction}

That medicaments can be absorbed through the skin has been known for a long time. While this route is usually of little significance percutaneous absorption of potent compounds can be important. The application of corticosteroid ointments and creams only permits an approximate dosage per unit area. An alternative approach is to use a range of steroid preparations of differing potency, so that an appropriate strength may be selected, depending on the diagnosis or severity of the disease.

The prolonged application of any potent corticosteroid may lead to atrophic skin changes and there is a risk of at least transient suppression of hypothalamic-pituitary-adrenal (H.P.A.) function. Thus, a corticosteroid with greater topical antiinflammatory activity than hydrocortisone but lesser potential for inducing side effects than the more potent compounds is needed. We describe here studies performed with a new glucocorticoid-clobetasone butyrate-to investigate its antiinflammatory activity and effect on H.P.A. function in patients with skin diseases. The use of an animal model to assess atrophic potential is also described.

\section{St. Bartholomew's Hospital, London EC1A 7BE}

D. D. MUNRO, M.D., F.R.C.P., Consultant Dermatologist

Glaxo Laboratories Ltd, Greenford, Middlesex

LYN WILSON, M.P.S., Clinical Research Unit Head

\section{Studies}

THE DRUG

Clobetasone butyrate is 21-chloro-11 dehydro betamethasone 17butyrate. It was selected for study because screening tests indicated a separation of topical from systemic activity. The comparative values for topical and systemic activity ${ }^{1}$ of clobetasone butyrate and five other steroids are given in the table (in arbitrary units).

Comparative Topical (Vasoconstriction) and Systemic (Thymus Involution) Activities of Six Corticosteroids

\begin{tabular}{l|c|c}
\hline \multicolumn{1}{c|}{ Corticosteroid } & $\begin{array}{c}\text { Vasoconstriction } \\
\text { Activity* }\end{array}$ & $\begin{array}{c}\text { Thymus Involution } \\
\text { Activity }\end{array}$ \\
\hline Betamethasone alcohol & $0 \cdot 8$ & 100 \\
Fluocinolone acetonide & 100 & 225 \\
Betamethasone valerate & 360 & 170 \\
Betamethasone behzoate & 345 & 829 \\
Clobetasol propionate & 1869 & 636 \\
Clobetasone butyrate & 263 & 9 \\
\hline
\end{tabular}

* On intact human skin.

† Subcutaneously in the mouse.

EFFECTIVENESS IN ECZEMA AND PSORIASIS

The topical anti-inflammatory activity of clobetasone butyrate was studied in a series of trials involving $\mathbf{4 0 9}$ patients with eczema. The steroid was formulated as a bland cream, without the addition of penetrants such as propylene glycol. Patients had bilateral, approximately symmetrical lesions to which the test and control preparations were allocated randomly; thus the patients acted as their own controls. All the trials were double blind, using the method described by Williams et al. ${ }^{2}$ Occlusive dressings were not used. Clobetasone butyrate was tested in increasing concentrations of $0.01 \%, 0.025 \%$ and $0.05 \%$ against hydrocortisone $1 \%$ cream.

The results of these dose response trials are shown in fig. 1. Clobetasone butyrate at $0.01 \%$ was similar to hydrocortisone $1 \%$, at $0.025 \%$ it was rather more effective, and at $0.05_{\%}^{\circ}$ it was significantly more effective $(P<0.05)$.

Though clobetasone butyrate $0.05 \%$ was not envisaged as being a primary treatment for psoriasis, which normally responds adequately only to the more potent steroids, we thought it appropriate to confirm the findings in eczema in patients with psoriasis. Differences in the relative activity of topical steroids are more readily shown in psoriasis, ${ }^{3}$ and hydrocortisone has little effect. Twelve patients with psoriasis were studied in a double-blind trial, using clobetasone butyrate $0.05 \%$ in a simple paraffin ointment base under occlusive dressings. Ten patients responded better to the clobetasone butyrate and two to the hydrocortisone ointment. Though the new steroid was more effective than hydrocortisone, the psoriasis did not respond to it as well as would have been expected had the condition been treated with betamethasone valerate or clobetasol propionate.

The activity of clobetasone butyrate $0.05^{\circ}{ }_{\circ}^{\prime}$ cream and ointment was further investigated in double-blind comparisons with fluocortolone 


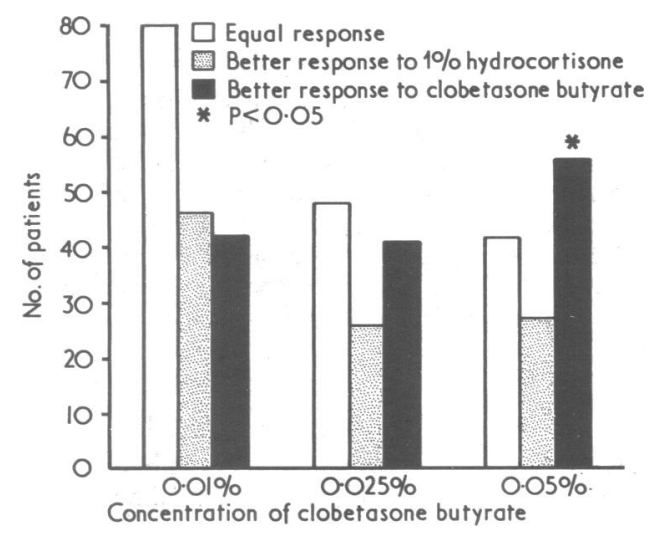

FIG. 1-Comparative response of patients with bilateral eczema to hydrocortisone $1 \%$ and clobetasone butyrate creams.

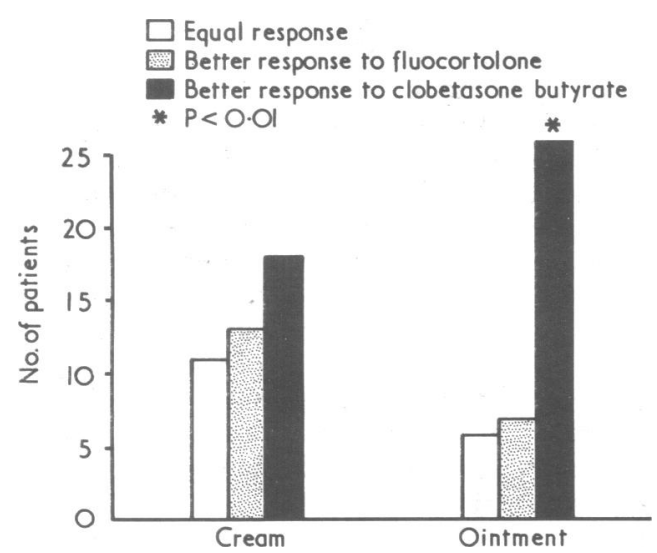

FIG. 2-Comparative response of patients with bilateral eczema to fluocortolone $0 \cdot 2 \%$ (Ultradil) and clobetasone butyrate $0.05 \%$ (Molivate).

pivalate and hexanoate ointment and cream $0.2 \%$ (Ultradil) in patients with bilateral eczema. Occlusive dressings were not used. The lesions treated with clobetasone butyrate responded better than those treated with fluocortolone (fig. 2), and the difference between the two ointments was statistically significant $(P<0 \cdot 01)$.

\section{EFFECT ON H.P.A. FUNCTION}

The effect of percutaneously absorbed betamethasone valerate on H.P.A. function has been described, ${ }^{4}$ and several other corticosteroids have been studied similarly (D. D. Munro, unpublished data). The effect of percutaneously absorbed clobetasone butyrate on the H.P.A. axis was investigated using the same protocol.

Adult patients whose inflammatory skin disease affected at least half of the body surface and whose condition warranted their admission to hospital were studied. For two or three days after admission the skin was treated only with bland ointment under stockinette dressings while baseline (9 a.m.) plasma corticosteroids and 24-hour urinary oxogenic steroids were measured. The integrity of the H.P.A. axis was tested by means of an insulin stress test (I.S.T.) except in one case when it was thought unjustifiable and a short tetracosactrin (Synacthen) test (S.T.T.) was substituted. Treatment with clobetasone butyrate ointment was then begun. Each day $30 \mathrm{~g}$ of ointment was applied and the body, including hands and feet, was totally occluded with polythene for 20 of the 24 hours. The active treatment period was 14 days for all except one patient, who received only 9 days' treatment. During the treatment period plasma and urinary corticosteroids were measured daily. At the end of the active treatment period the patient was given only bland ointment for two or three further days while the tests of H.P.A. function were repeated.

Two groups of patients were studied. Six patients were treated with clobetasone butyrate $0 \cdot 1 \%$ ointment, which is twice the concentration of the commercial preparation. During the treatment period the plasma corticosteroid levels fell in three of the patients and remained virtually normal in the other three. The urinary oxogenic steroid levels, though a little reduced during treatment in four patients, were not significantly different from pretreatment values. The pretreatment I.S.T. and S.T.T. results were normal. The results (I.S.T.) after treatment were abnormal in three cases, though only marginally so in two of them.

The second group comprised three patients treated with clobetasone butyrate $0.05 \%$ ointment (Molivate). Two patients had atopic eczema affecting $100 \%$ and $90 \%$ of body surface, and the third had constitutional eczema covering $60 \%$. All pretreatment and posttreatment I.S.T. and S.T.T. results were normal. The third patient showed no variation in daily plasma corticosteroid levels during active treatment. In the patient affected over $90 \%$ of his body surface the plasma and urinary corticosteroids remained within normal limits though there were minimal falls of both levels in the middle of treatment (fig. 3). The remaining patient had similar mid-treatment falls but to a greater degree. In both these patients the levels recovered while treatment continued. All three patients responded to treatment and their skin was free of rash by the end of the study. Healing was slower than might have been expected with more potent corticosteroids.

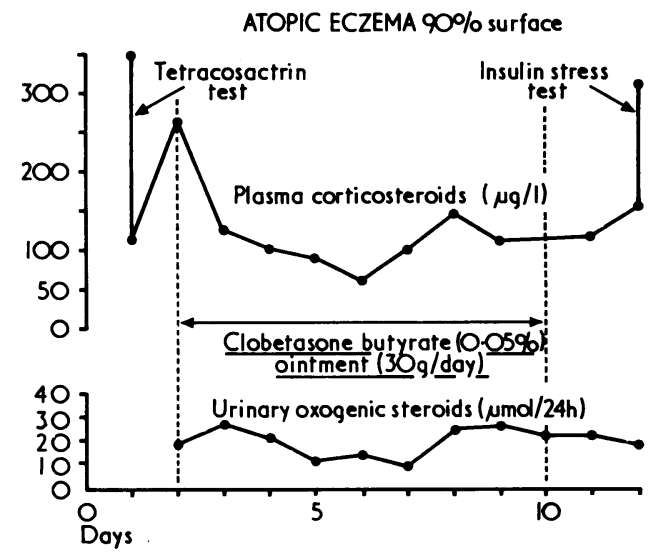

FIG. 3-Plasma corticosteroid and urinary oxogenic steroids before, during, and after treatment with clobetasone butyrate $0.05 \%$ ointment under polythene occlusion in one patient.

Conversion: SI to Traditional Units-Plasma corticosteroids: $1 \mu \mathrm{g} / 1=0 \cdot 1 \mu \mathrm{g} / 100 \mathrm{ml}$ (normal range 60 $220 \mu \mathrm{g} / \mathrm{l})$. Oxogenic steroids: $1 \mu \mathrm{mol} / 24 \mathrm{~h} \approx 0.29$ $\mu \mathrm{g} / 24 \mathrm{~h}$.

\section{EFFECT ON EPIDERMIS}

A method of inducing epidermal thinning in pigs by the application of corticosteroids has been described by Winter and Wilson. ${ }^{5}$ The formulated steroidal compounds were applied to the shaved dorsal skin of the large white domestic pig. Standard quantities packed in coded identical tubes were spread under occlusive dressings on five days of each week for seven weeks. Unmedicated ointment or cream base and hydrocortisone $1 \%$ were used as controls. Biopsies were performed before and throughout treatment. Before the code was broken the relative effect of various corticosteroids on epidermal thickness was judged qualitatively by histological appearance and quantitatively by projecting an image of a cross-section on paper and measuring the area of the epidermis with a planimeter.

Clobetasone butyrate $0.05 \%$ was compared in this test with five other topically active corticosteroid ointments. The six fell into three groups: those with a pronounced effect-flurandrenolone $0.05 \%$ and fluocinolone acetonide $0.025 \%$; those with a slight to moderate effect-triamcinolone acetonide $0 \cdot 1 \%$, flurandrenolone $0.0125 \%$, and clobetasone butyrate $0.05 \%$; and those with little or no effectunmedicated base and hydrocortisone $1 \%$. Clobetasone butyrate ointment caused the least epidermal thinning among the slight-tomoderate group. The detailed results of this study are described elsewhere. ${ }^{6}$

\section{Discussion}

As ointment and cream at a concentration of $0.05 \%$ clobetasone butyrate has useful topical anti-inflammatory activity. In conventional double-blind comparative trials it treated eczema 
more effectively than hydrocortisone $1 \%$ or fluocortolone pivalate and hexanoate $0.2 \%$. Of the 212 patients treated with the definitive formulations about a third were children under the age of six years. No untoward effects have been reported.

The tests of the effect of percutaneously absorbed clobetasone butyrate on H.P.A. function were carried out under conditions which predisposed to maximum absorption-namely, extensive disruption of the cutaneous barrier, the application of large quantities of ointment, and hydration of the skin by virtually continuous whole-body occlusion. The minimal effect observed was in definite contrast to that seen with other preparations. Betamethasone valerate caused H.P.A. axis suppression in all patients tested, ${ }^{4}$ and, though in one or two the corticosteroid levels showed a tendency to rise again during treatment as the skin healed, full recovery was not seen until after treatment had ceased. In a similar study patients treated with clobetasol propionate remained suppressed throughout the period of active treatment (D. D. Munro, unpublished data), and there is evidence that this potent compound can cause H.P. A.suppression in ordinary clinical use, especially when the amount applied to the skin is excessive. ${ }^{78}$ This principle applies to all potent corticosteroid ointments whether or not they contain halogen atoms. In a further similar study of four patients using hydrocortisone butyrate cream $0.1 \%$-a preparation claimed to be "as safe as hydrocortisone"-comparison of 50 plasma cortisol levels during treatment with 15 pretreatment and 11 posttreatment readings showed suppression of plasma cortisol ( $P<0.001$ and $P<0.05$, respectively, for pretreatment $v$. during treatment and during treatment $v$. posttreatment). In contrast, three of the six patients on whom clobetasone butyrate $0.1 \%$ was used showed no suppression. Of the three patients treated with the ointment at half this strength, $0.05 \%$ (Molivate), two had normal values throughout and the third, whose total body surface was affected, had low levels churing the first few days of treatment, but these became normal again while treatment continued.

The rigorous conditions of this test are rarely met in clinical practice. Clobetasone butyrate preparations have been designed for use in less severe cases and, rather than use them in such a regimen, a short course of a more potent steroid would usually be preferred. Clobetasone butyrate is unlikely to have any effect on H.P.A. function used in the manner for which it is intended. Even hydrocortisone, however, applied in excessive amounts in infants, may cause adverse systemic effects, 910 which must be borne in mind when treating such vulnerable patients.

More of a problem with topical steroids are local skin changes. Atrophy and striae are usually the result of prolonged use, often with occlusive dressings or in flexures, while changes on the face arise most often from the inappropriate prescribing of steroids for acne or rosacea. While these effects are probably avoidable, even in the management of chronic conditions, it would be an advantage to be able to offer a treatment which would control the patient's disease yet be less likely to cause thinning of the skin.

The skin of the young domestic pig has been used extensively in wound-healing studies and in many respects behaves like that of man.11 Winter and Wilson ${ }^{5}$ have described the epidermal thinning which topical corticosteroids can induce in pig skin, showing that different steroid preparations cause different degrees of thinning. Clobetasone butyrate $0.05 \%$ applied under occlusion for seven weeks caused much less epidermal thinning in the pig than flurandrenolone $0.05 \%$ or fluocinolone acetonide $0.025 \%$, both of which caused severe atrophy. Triamcinolone acetonide $0.1 \%$, flurandrenolone $0.0125 \%$, and clobetasone butyrate $0.05 \%$ caused moderate to slight atrophy-the clobetasone butyrate preparation having the least effect of the three.

Clinically obvious atrophy induced by topical corticosteroids involves dermal as well as epidermal changes. In the pig dermal changes are difficult to quantify. Corticosteroids which eventually cause dermal thinning may reasonably be assumed first to cause changes in the epidermis. A lesser effect on the outer structures probably indicates that there would be a delay in the appearance of changes in the dermis.

As with an effect on pituitary adrenal function, so an effect on collagen seems to be inseparable from glucocorticoid antiinflammatory activity. Though hydrocortisone has not been shown to cause thinning of the skin in the pig, and atrophy after its topical use is not seen in patients, the high circulating cortisol levels found in Cushing's syndrome are associated with atrophic skin changes.

Conclusions.-Clobetasone butyrate $0.05 \%$ is a new corticosteroid with topical anti-inflammatory activity greater than hydrocortisone $1 \%$ and fluocortolone $0.2 \%$. Under the most stringent conditions its effect on H.P.A. function was minimal and in an animal model it caused less epidermal thinning than the other steroids tested, with the exception only of hydrocortisone. Such a combination of clinical activity with a wide margin of safety should make clobetasone butyrate a useful preparation for the management of the more tractable inflammatory dermatoses or where a high ratio of safety to efficacy is required. It also provides the means of a more logical approach to the management of chronic conditions by enabling courses of the more potent corticosteroids necessary to control severe exacerbations to be alternated with maintenance treatment that is less likely to give rise to problems.

We thank the following dermatologists who contributed cases for the clinical trials reported: Professors L. Juhlin and B. Magnusson and Drs. L. Gip and K. Hersle, Sweden; Dr. J. Van der Meersch, Belgium; Dr. W. A. Birkett, Hastings; Dr. A. Bowyer, Torbay; Dr. M. Hewitt, Truro; and Dr. C. J. Stevenson, Newcastle. Finally, we thank Dr. G. D. Winter of the Institute of Orthopaedics, Stanmore, for allowing us to quote from his unpublished work.

\section{References}

1 Child, K. J., et al., Archives of Dermatology, 1968, 97, 407.

2 Williams, D. I., et al., Lancet, 1964, 1, 1177.

3 Sparkes, C. G., and Wilson, L., British fournal of Dermatology, 1974, 90, 197.

4 James, V. H. T., Munro, D. D., and Feiwel, M., Lancet, 1967, 2, 1059.

5 Winter, G. D., and Wilson, L., in Mechanisms of Topical Corticosteroid Activity. London, Churchill Livingston, in press.

6 Winter, G. D., and Wilson, L., British fournal of Dermatology, in press.

${ }^{7}$ Staughton, R. C. D., and August, P. J., British Medical fournal, 1975, 2, 419.

${ }^{8}$ Tan, R. S. H., Proceedings of the Royal Society of Medicine, 1974, 67, 719.

Benson, P. F., and Pharoah, P. O. D., Guy's Hospital Reports, 1960, 109, 212.

${ }^{10}$ Feinblatt, B. I., et al., American fournal of Diseases of Children, 1966, 112, 218.

11 Winter, G. D., in Epidermal Wound Healing, ed. H. I. Maibach and D. T. Rovee, p. 71. Chicago, Year Book Medical Publishers Inc., 1972. 